\title{
Measurements and Analysis of Microwave Nonlinearities in Ferroelectric Thin Film Transmission Lines
}

\author{
J. Mateu', J. C. Booth', S. A. Schima', C. Collado', D. Seron², J.M. O'Callaghan ${ }^{2}$ \\ ${ }^{1}$ National Institute of Standards and Technology, Boulder, CO, 325 Broadway/80305, USA \\ ${ }^{2}$ UPC (Universitat Politècnica de Catalunya), C/ Jordi Girona, Barcelona 08034, Spain
}

\begin{abstract}
This work evaluates the microwave nonlinear properties of ferroelectric BaSrTio thin films by measuring the frequency response of several coplanar transmission lines and interdigital capacitor structures as a function of the applied electric field from $150 \mathrm{~Hz}$ to $40 \mathrm{GHz}$. From these measurements, we obtain the distributed nonlinear capacitance $C\left(V_{d c}\right)$ as a function of dc bias. We also measure the harmonic generation at microwave frequencies in ferroelectric transmission lines, and use an accurate circuit model to obtain $C\left(V_{r f}\right)$, the nonlinear capacitance as a function of $r$ bias. Information about the tuning speed of the film is obtained from a comparison between the two nonlinear capacitances. Characterization of this mechanism is also required to assess the spurious signal generation in ferroelectric-based devices.
\end{abstract}

Index Terms - Ferroelectric films, microwave measurements, nonlinearities, circuit modeling, Harmonic Balance.

\section{INTRODUCTION}

Ferroelectric thin film materials are important for many microwave applications such as those requiring frequencyagility, phase shifting, harmonic generation or pulse shaping [1]. The combination of these materials with high temperature superconductors (HTS) is being considered to achieve lowloss filters with electronic frequency tuning/trimming features [2], or to reduce the nonlinear effects inherent to HTS components [3].

The applications above are based on the possibility of changing the permittivity of the material with an applied electric field. A detailed understanding and a broadband characterization of this mechanism are required to assess critical issues such as maximum tuning speed or spurious signal generation in ferroelectric-based devices.

This work presents measurements and analysis of the microwave nonlinear response in a $400 \mathrm{~nm} \mathrm{Ba}_{0.3} \mathrm{Sr}_{0.7} \mathrm{TiO}_{3}$ (BSTO) thin film grown by pulsed laser deposition on a $16 \times 16$ $\mathrm{mm}^{2} \mathrm{LaAlO}_{3}(\mathrm{LAO})$ substrate. The device geometries that are patterned onto the film under test and the measurement procedure used to evaluate these structures are described in Section II. Broadband microwave and third harmonic measurements are reported and analyzed in Sections III and IV, respectively. Comparison between these measurements is discussed in Section V.

\section{SAMPLES AND MeAsuremENTS}

The film under test is patterned with coplanar waveguide (CPW) transmission lines and interdigital capacitors (IDC) by using lithographic lift-off procedures with $0.3 \mu \mathrm{m}$-thick Au conductors deposited by electron beam evaporation. The CPW transmission lines and IDCs have a center conductor linewidth of $20 \mu \mathrm{m}$. Three different sets of devices are fabricated with gaps between the center conductor of the transmission lines and their ground planes of 10,20 and $40 \mu \mathrm{m}$. The same gaps define the spacing between the fingers of the IDC structures. The lengths of the lines are $0.42,2.15,3.22,5.93,12.57 \mathrm{~mm}$, and we refer to these lines as $\mathrm{L}_{1}, \mathrm{~L}_{2}, \mathrm{~L}_{3}, \mathrm{~L}_{4}$ and $\mathrm{L}_{5}$, respectively. The effective lengths of the IDC structures (i.e., the length where the fingers overlap) are $0.1,1.83,2.9,5.61,12.25 \mathrm{~mm}$, respectively. Identical structures are also patterned in bare LAO substrate. Also identical measurements are performed on the bare LAO substrate structures in order to obtain the background contributions to the measurements of the film under test.

The dc-biased measurements consist of room-temperature, on-wafer measurements on patterned planar devices as a function of frequency from $150 \mathrm{~Hz}$ to $40 \mathrm{GHz}$. To cover the whole frequency range we used several measurement techniques to measure a variety of CPW transmission lines and IDCs on the test sample and also on the bare substrate. We use multiline thru-reflect-line (TRL) calibration for CPW transmission lines to obtain the complex propagation constant [4]. By using accurate distributed circuit models for both CPW lines and IDCs, we determine the capacitance and conductance per unit length as a function of frequency. These data are then reduced to a single, geometry-independent parameter (the complex permittivity) at each frequency point. This is accomplished using a 2D finite-element electromagnetic simulator to calculate the capacitance per unit length as a function of film permittivity for different cross sections of IDCs and CPW lines. This procedure is performed as a function of the applied electric field by applying a bias voltage $\left(\mathrm{V}_{\mathrm{dc}}\right)$ through a bias tee operating up to $40 \mathrm{GHz}$. We then obtain the capacitance $\left(C\left(V_{d d}\right)\right)$ and permittivity as a function of applied dc voltage. 
The procedure for the rf-biased measurements includes the measurement of the third harmonic and intermodulation product (IMD) generated when the CPW transmission lines are fed with one or two tones, respectively. These nonlinear effects come from the dependence of the capacitance per unit length [5] as a function of the applied rf voltage, $C\left(V_{t f}\right)$. The aim of these measurements is two-fold. On one hand, by comparing $C\left(V_{d t}\right)$ and $C\left(V_{d d}\right)$ we can evaluate the attainable time scales for tuning [5]. One the other hand, modeling the nonlinear effects in devices at these frequencies is important in order to assess their generation of passive IMD and other spurious signals, and evaluate the possible role of ferroelectric materials in the compensation of the nonlinear effects produced by HTS materials [3]. The full determination of the nonlinear behavior should include harmonic measurement for several frequencies of the fundamental tone and IMD measurements performed as a function of the spacing between the input tones. This report includes the third harmonic measurements at a fixed fundamental frequency.

\section{Broadband Microwave Measurements}

The microwave properties of the BSTO film have been determined by using three different measurement set-ups that cover the frequency range from $150 \mathrm{~Hz}$ to $40 \mathrm{GHz}$. By using an LCR meter we measure the $150 \mathrm{~Hz}$ to $1 \mathrm{MHz}$ frequency range. An rf network analyzer performs the measurements from $300 \mathrm{kHz}$ to $200 \mathrm{MHz}$. Finally a high-frequency network analyzer is used to cover the range from $10 \mathrm{MHz}$ to $40 \mathrm{GHz}$. We refer to these sets as low frequency (LF), intermediate frequency (IF) and high frequency (HF) measurements, respectively. Measurements at LF and IF are performed on both CPW transmission lines and IDC structures. Since at these frequencies these structures behave as lumped elements, we use their frequency response to directly obtain the

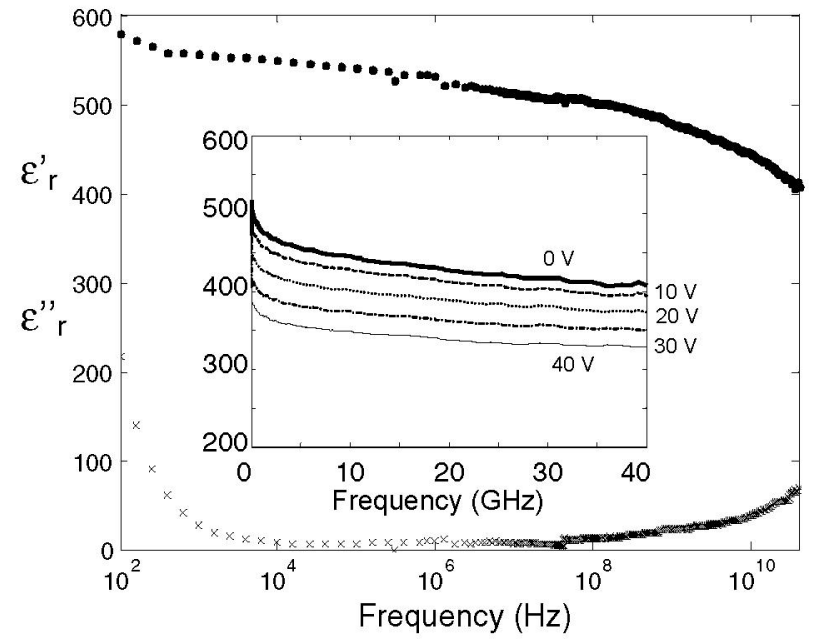

Fig. 1. BSTO thin-film real (dots) and imaginary (crosses) part of the permittivity from $150 \mathrm{~Hz}$ to $40 \mathrm{GHz}$. Frequency axis in $\log$ scale. Inset: Real part of the permittivity as a function of the bias voltage. Thick, dashed, dotted, dot-dashed and thin lines correspond to $0,10,20,30$ and $40 \mathrm{~V}$, respectively. capacitance to the ground and the series capacitance, respectively. In contrast, at $\mathrm{HF}$ we only measure the response of the CPW transmission lines. From the complex propagation constant obtained from the TRL calibrations of the transmission lines, we find the capacitance and conductance per unit length. The distributed parameters extracted from the measurement are then used to obtain the real and imaginary part of the thin-film permittivity as a function of frequency; i.e., $\varepsilon^{\prime},(f)$ and $\varepsilon^{\prime \prime},(f)$.

As mentioned above, we applied this procedure as a function of the bias voltage from 0 to $40 \mathrm{~V}$ in all structures. Note that this gives different values for the electric field in the structures according to the gap spacing between the conductor and the ground.

Fig. 1 outlines $\mathcal{E}_{r}^{\prime}(f)$ and $\mathcal{E}^{\prime \prime},(f)$ at $0 \mathrm{~V}$ bias voltage. They are represented by dots and crosses, respectively. The imaginary part of the permittivity increases as the frequency decreases below approximately $10 \mathrm{kHz}$. This is due to dc conductivity. The inset of Fig. 1 depicts the frequency dependence of the real part of permittivity for several bias voltages. Thick-solid, dashed, dotted, dot-dashed and thin lines correspond to 0,10 , 20,30 and $40 \mathrm{~V}$, respectively. These results are consistent with those given in the literature [6].

To quantify the deviation of $\varepsilon_{r}^{\prime}$ caused by the electric field (or applied voltage) we define:

$$
\Delta \varepsilon_{r}^{\prime}(E)=\left[\varepsilon_{r}^{\prime}(E)-\varepsilon_{r}^{\prime}(0)\right] / \varepsilon_{r}^{\prime}(0),
$$

where $\varepsilon_{r}^{\prime}(E)$ is the permittivity at a given electric field and $\varepsilon_{r}^{\prime}(0)$ the permittivity at $0 \mathrm{~V} / \mathrm{m}$ electric field. Fig. 2 plots $\Delta \mathcal{E}_{r}^{\prime}(E)$ obtained from the measurements in transmission lines with 10,20 and $40 \mu \mathrm{m}$ gap, represented by squares, crosses and circles, respectively. Note that the variation observed for the different gaps shows excellent agreement. At low fields we can fit a square-law dependence with electric field, that is $\Delta \mathcal{E}_{r}$,

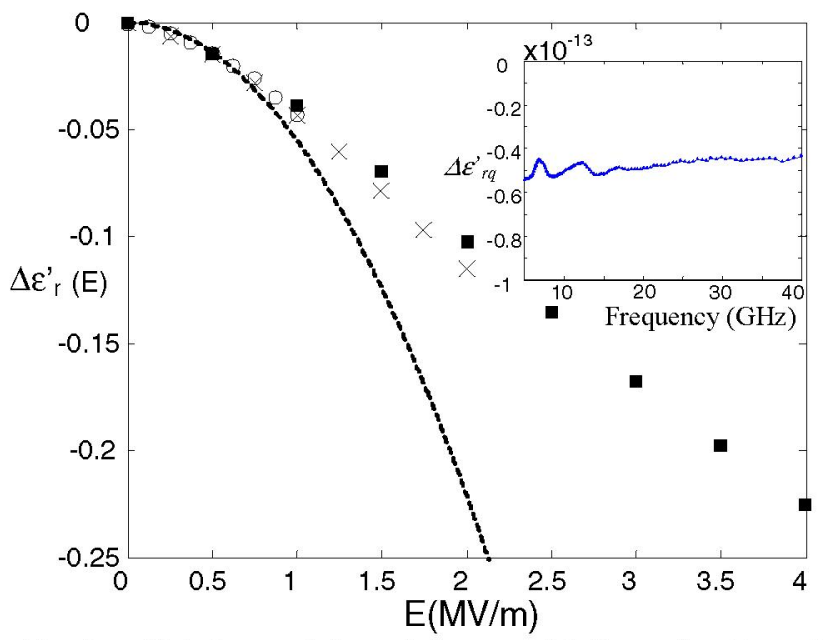

Fig. 2. Relative variation of the permittivity as function of the applied electric field at $2 \mathrm{GHz}$. Squares, crosses, circles obtained from 10,20 and $40 \mu \mathrm{m}$ gap transmission lines, respectively. Dashed line fitting by using a square dependence approximation. Inset: Square dependence term, $\Delta \varepsilon_{r q}^{\prime}$, from $10 \mathrm{MHz}$ to $40 \mathrm{GHz}$. 
$(E)=\Delta \mathcal{E}_{r q}^{\prime} \cdot E^{2}$. The inset of Fig. 2 shows $\Delta \mathcal{E}_{r q}^{\prime}$ as a function of frequency. Note that it remains fairly constant over the whole frequency range.

\section{THIRd Harmonic MEASUREMENTS In TRAnSMISSION LINES}

To address the nonlinear response of the BSTO thin films for a fast input signals (nanosecond time scale), we measured the third harmonic when the transmission lines under test are fed with a fundamental signal at $2 \mathrm{GHz}$. Nonlinear measurements on the bare LAO substrate sample have also been performed in order to characterize the nonlinear effects coming from the measurement setup.

Results of those measurements for $20 \mu \mathrm{m}$ gap transmission lines are shown in Fig. 3. This figure represents the third harmonic output power (in $\mathrm{dBm}$ ) as a function of the output power of the fundamental (in $\mathrm{dBm}$ ). Circles, triangles-down, squares, triangles-up and diamonds correspond to $\mathrm{L}_{5}, \mathrm{~L}_{4}, \mathrm{~L}_{3}, \mathrm{~L}_{2}$ and $\mathrm{L}_{1}$, respectively. These results show how the third harmonic increases proportionally with the length of the transmission line, which is consistent with the distributed origin of the nonlinear effects due to the dependence of the distributed capacitance on the applied voltage $C\left(V_{r f}\right)$ [5]. Although a decrease in the slope occurs at high powers due to heating effects, the third harmonic (on a logarithmic scale) follows a slope 3 dependence on the fundamental tone (on a logarithmic scale) over most of the measurement range. This suggests a square law dependence between the distributed capacitance and $V_{r f}$ :

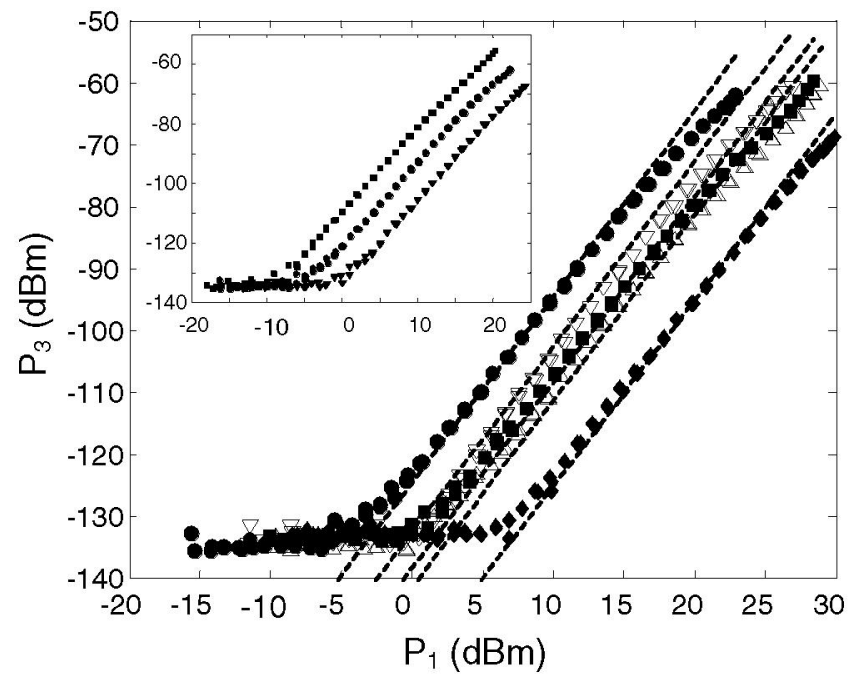

Fig. 3. Output power of the third harmonic as a function of the output power of the fundamental for transmission lines of $20 \mu \mathrm{m}$ gap. Circles, triangles-down, squares, triangles-up and diamonds correspond to $\mathrm{L}_{5}, \mathrm{~L}_{4}, \mathrm{~L}_{3}, \mathrm{~L}_{2}$ and $\mathrm{L}_{1}$, respectively. Dashed lines correspond to the simulated results. Inset: Output power of the third harmonic as a function of the power of the fundamental for $\mathrm{L}_{5}$, with 10 (squares), 20 (circles) and 40 (triangles) $\mu \mathrm{m}$ gap.

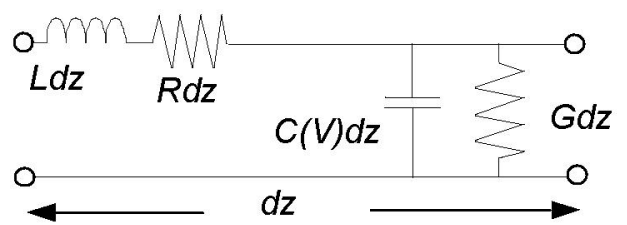

Fig. 4. Elemental section of a transmission line of $d z$ length with a nonlinear dielectric substrate.

$$
C\left(V_{r f}\right) \approx C_{0} \cdot\left(1+\Delta C_{2_{-} r f} \cdot V_{r f}^{2}\right),
$$

where $C_{0}$ is the linear part of the capacitance and $\Delta C_{2, f}$ is the coefficient accounting for the quadratic rf voltage dependence. Note that the term $\Delta C_{2-f} \cdot V_{r f}^{2}$ defines the relative variation of the capacitance with of voltage.

To obtain $\Delta C_{2}$ of we fitted the measured third harmonic to that produced by a nonlinear transmission line whose elemental segment is shown in Fig. 4. Detailed analysis of this circuit model, for matched transmission lines, is presented in [3], where closed-form expressions connecting the third harmonic (and also IMD) with the nonlinear distributed parameters $\left(C\left(V_{t}\right)\right.$ in this case $)$ can be found.

Since we performed nonlinear measurements on transmission lines with several gaps and equal center conductor linewidths, having different impedances, we may not apply the closed-form solution reported in [3]. We used a commercial software circuit analysis to implement the circuit model, which can be solved using Harmonic Balance techniques [7]. The linear part of the distributed parameters characterizing the elemental segment of Fig. 4 is obtained from the measurements reported in Section III. Note that accurate modeling of the transmission lines at the fundamental frequency $(2 \mathrm{GHz})$ and its harmonics requires us to explicitly consider the frequency dependence of the linear part of the distributed transmission line parameters.

By using the circuit model of Fig. 4 and the nonlinear dependence of (2) a unique value of $\Delta C_{2, f}$ is used to fit the measurements of Fig. 3. Dashed lines in Fig. 3 indicate the results of the simulation, showing very good agreement with the measurements for the different transmission lines length, for a single value of $\Delta C_{2,5}$

The inset of Fig. 3 compares the third harmonic measured on $\mathrm{L}_{5}$ for 10,20 and $40 \mu \mathrm{m}$ gap, represented by squares, circles and triangles, respectively. As we might expect, the nonlinear effects increase as the gap decreases. Values of $\Delta C_{2, f}$ obtained from the measurements are summarized in the fourth column of Table I. Note that these values are scaled by the gap to the power of 2 .

\section{DISCUSSION}

The nonlinear distortion effects obtained and discussed from third harmonic measurements in Section IV result in a nonlinear circuit distributed term, $\Delta C_{2 \_f}$. Scaling this term by the gap squared results in a consistent nonlinear term for 
TABLE I

SUMMARY OF NONLINEAR C AND $\varepsilon_{r}^{\prime}$

\begin{tabular}{|c|c|c|c|c|}
\hline $\begin{array}{c}\text { Gap } \\
(\mu \mathrm{m})\end{array}$ & $\mathrm{C}_{0}(\mathrm{~F} / \mathrm{m})$ & $\begin{array}{c}\Delta \mathrm{C}_{2} \text { dc } \\
(\mu \mathrm{m} / \mathrm{N})^{2}\end{array}$ & $\begin{array}{c}\Delta \mathrm{C}_{2} \text { rf } \mathrm{g}^{2} \\
(\mu \mathrm{m} / \mathrm{N})^{2}\end{array}$ & $\begin{array}{c}\Delta \varepsilon_{\text {rq }}^{\prime} \\
(\mathrm{m} / \mathrm{V})^{2}\end{array}$ \\
\hline $\mathrm{g}=10$ & $7.06 \cdot 10^{10}$ & -0.0183 & -0.00141 & $5.12 \cdot 10^{-14}$ \\
\hline $\mathrm{g}=20$ & $4.73 \cdot 10^{10}$ & -0.0178 & -0.00138 & $5.01 \cdot 10^{-14}$ \\
\hline $\mathrm{g}=40$ & $3.31 \cdot 10^{10}$ & -0.0180 & -0.00152 & $5.52 \cdot 10^{-14}$ \\
\hline
\end{tabular}

transmission lines with different gaps. Note that this is very important since we could also predict the nonlinear response in future devices with different geometries than the ones measured.

The nonlinear term $\Delta C_{2} r$, also quantifies the variation of the permittivity when the applied electric field in the transmission line changes on a nanosecond time scale. To determine if the device can achieve full tunability at these time scales we compared the variation of the capacitance obtained in Section IV, $C\left(V_{r t}\right)$, with the one obtained from measurements in Section III, $C\left(V_{d c}\right)$. To do so we define $C\left(V_{d c}\right)$ as:

$$
C\left(V_{d c}\right) \approx C_{0} \cdot\left(1+\Delta C\left(V_{d c}\right)\right),
$$

where $C_{0}$ is $\mathrm{C}\left(\mathrm{V}_{\mathrm{dc}}=0\right)$ and the term $\Delta C\left(V_{d c}\right)$ is the relative variation of the capacitance with dc voltage. Note that $\Delta C\left(V_{d c}\right)$ is analogous to $\Delta \mathcal{E}_{r}^{\prime}(E)$ defined in (1), the quadratic coefficient of which $\left(\Delta \mathcal{E}_{r q}^{\prime}(E)\right)$ is also reported in Table I. To compare (2) and (3) we also use a square law dependence to fit the measurements of $C\left(V_{d c}\right)$. That is $\Delta C\left(V_{d c}\right)=\Delta C_{2 \_d c} \cdot V_{d c}^{2}$.

Fig. 5 shows the measured $\Delta C\left(V_{d c}\right)$ at $2 \mathrm{GHz}$, scaled by the square of the gap. Squares, crosses and circles correspond to the measurements in 10,20 and $40 \mu \mathrm{m}$ gap transmission lines. This figure also shows the fitting using (3), represented by thin lines. The coefficient $\Delta C_{2} d c^{\prime} g^{2}$ resulting from these fits is summarized in the third column of Table I. Note that in this case we also obtain a consistent value for different values of the transmission line gaps. Fig. 5 also plots the term $\Delta C_{2 \text { rf }} g^{2} V^{2}$, represented by dashed lines. By comparing the results in Fig. 5 or the terms $\Delta C_{2, r f} g^{2}$ and $\Delta C_{2 d i} \cdot g^{2}$ of table I we conclude that full tunability cannot be achieved in these devices at nanosecond time scales.

To accurately predict for what time scales full tunability can be achieved, further experiments are required. We plan to repeat the third harmonic measurements for several values of the fundamental frequency as well as use IMD measurements of the nonlinearities as a function of the spacing between tones [8].

\section{CONCLUSIONS}

This work described the procedure for characterizing the microwave properties of ferroelectric thin-films using

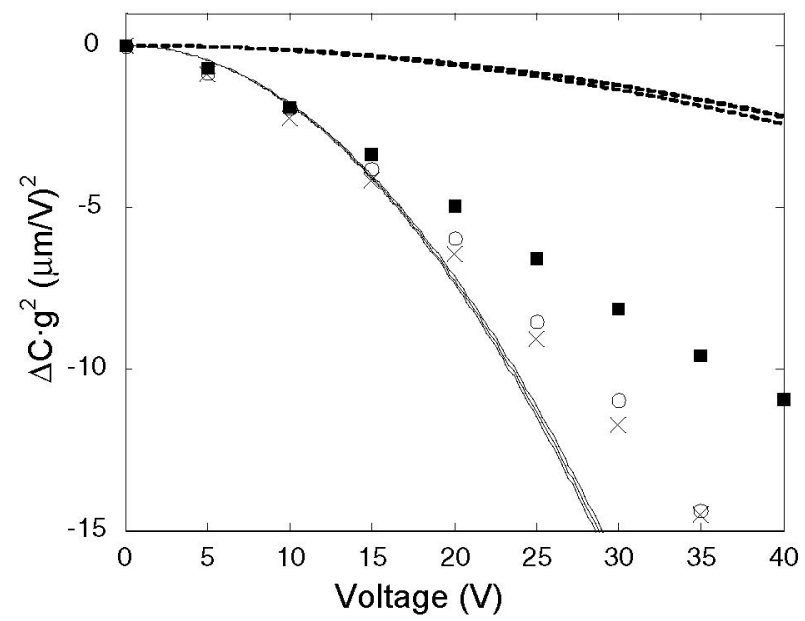

Fig. 5. Square, cross and circles: measured relative variation of the capacitate for 10,20 and $40 \mu \mathrm{m}$ gap transmission lines. Thinlines outline $\Delta \mathrm{C}_{2} \mathrm{dc} \cdot \mathrm{g}^{2} \cdot \mathrm{V}^{2}$ as a function of voltage. Dashed-lines outline $\Delta \mathrm{C}_{2} \mathrm{rf} \mathrm{g}^{2} \cdot \overline{\mathrm{V}}^{2}$ as a function of voltage.

coplanar microwave structures. Determination and modeling of the nonlinearities have been performed with third harmonic measurements. Comparison of the nonlinear response at different time scales has been analyzed by comparing circuit model parameters from dc bias measurements and third harmonic measurements. Identification of new experiments has been also reported.

\section{REFERENCES}

[1] M. J. Lancaster, J. Powel and A. Porch, "Thin-film ferroelectric microwave devices," Supercond. Sci. Technol. no. 11, pp. 13231334, November 1998.

[2] B. H. Moeckly, Y. Zhang, "Strontium Titanate Thin films for Tunable YBaCuO Microwave filters", IEEE Trans. Applied Supercond., Vol. 11, n.1, pp. 450-453, March 2001.

[3] D. Seron, C. Collado, J. Mateu and J.M. O'Callaghan, "Analysis and Simulation of Distributed Nonlinearities in Ferroelectrics and Superconductors for Microwave Applications," IEEE Trans. Microwave Theory \& Tech., vol. 54, no. 3, pp-1154-1160, 2006.

[4] R.B. Marks, "A Multiline method for Network analyzer calibration", IEEE Ttrans. Microwave Theory \& Tech. Vol. 39, no 7, pp. 1205-1215, July 1991.

[5] J. C. Booth and R. H. Ono, "Microwave frequency tuning and harmonic generation in ferroelectric thin film transmission lines," Appl. Phys. Lett., vol. 82, no. 4, pp. 718-720, July 2002.

[6] J.C. Booth, L.R. Vale and R. H. Ono, "Broadband Determination of Microwave Permittivity and loss in tunable dielectric thin film materials", Materials Issue for Tunable RF and Microwave Devices, Proceedings of the 1999 MRS Meeting, Vol. 603.

[7] S.A. Maas, Nonlinear Microwave Circuits, Artech House, 1988.

[8] T. Chakraborty, I. Hunter, R.Kurchania, A. Bell anc S. Chakraborty, "Intermodulation distortion in Wide-Band DualMode Bulk Ferroelectric Bandpass Filters" 2005 IEEE MTT-S Int. Microwave Symp. Dig., pp. 615-618, June 2005. 